\title{
Low-noise X-ray PIN photodiodes made of perovskite single crystals by Solution-processed doped epitaxial growth
}

Xin Wang ( $230159424 @ s e u . e d u . c n)$

Southeast University https://orcid.org/0000-0003-2496-2872

Yubing Xu

Southeast University

Yuzhu Pan

Southeast University

Yuwei Li

Southeast University

Ji Xu

Southeast University

Jing Chen

Southeast University

Jun Wu

Southeast University

Qing Li

Southeast University

Xiaobing Zhang

Southeast University

Zhiwei Zhao

Southeast University

Elias Elemike

Chemistry Department of North West University

Damian Onwudiwe

Chemistry Department of North West University

Javed Akram

COMSATS University Islamabad

Wei Lei

Southeast University 
Keywords: Low-noise photodiodes, metal halide perovskites (MHPs), perovskite single crystals, solutionprocessed doped epitaxial growth

Posted Date: October 7th, 2020

DOI: https://doi.org/10.21203/rs.3.rs-83921/v1

License: (c) (1) This work is licensed under a Creative Commons Attribution 4.0 International License. Read Full License 


\section{Low-noise X-ray PIN photodiodes made of perovskite single crystals by Solution- processed doped epitaxial growth}

Xin Wang1*, Yubing $\mathrm{Xu}^{1}$, Yuzhu Pan ${ }^{1}$, Yuwei $\mathrm{Li}^{1}$, Ji Xu${ }^{1}$, Jing Chen ${ }^{1}$, Jun $\mathrm{Wu}^{1}$, Qing $\mathrm{Li}^{1}$, Xiaobing Zhang ${ }^{1}$, Zhiwei Zhao ${ }^{1}$, Elias E. Elemike², Damian C. Onwudiwe², Javed Akram³ ${ }^{3}$, Wei Lei* ${ }^{1}$ Joint International Research Laboratory of Information Display and Visualization, School of Electronic Science and Engineering, Southeast University, Nanjing 210096, China

${ }^{2}$ Chemistry Department of North West University, Mafikeng, South Africa

${ }^{3}$ COMSATS University Islamabad, Islamabad, Pakistan

E-mail: 230159424@seu.edu.cn, lw@seu.edu.cn

X-ray photodiodes made of metal halide perovskites (MHPs) which directly convert X-ray photons into electron-hole pairs have shown advantages in low-cost and high X-ray detection sensitivity. However, devices fabricated by spin-coating and evaporation methods suffer from high traps density near poor interfacial layers (n-type/intrinsic and p-type/intrinsic) which lead to high dark current and noise current under large reverse bias. In this work, solutionprocessed doped epitaxial growth is employed to limit these traps through epitaxially growing n-type MHPs (bismuth-doped) and p-type MHPs (silver-doped) on opposite faces of intrinsic $\mathrm{CH}_{3} \mathrm{NH}_{3} \mathrm{PbBr}_{2.5} \mathrm{Cl}_{0.5}$ MHP single crystals. Through energy structure design, effective electron/hole blocking layers could decrease the noise and dark current, these X-ray PIN photodiodes work under a large external electrical field, which enables a state-of-art response speed (fall) of $750 \mathrm{~ns}$ and a lowest detectable dose rate of $17.7 \mathrm{nGys}^{-1}(40 \mathrm{kVp})$. This work will motivate new strategies to fabricate high-performance devices based on perovskites using solution-processed methods. These founding also explore a new generation of low dose and high dynamic X-ray detectors based on MHPs.

X-ray photodiodes, which directly convert X-ray photons into electron-hole pairs, play an increasingly important role in low-dose and high-resolution X-ray radiography. Besides CdZnTe single crystals $^{1}$, CdTe single crystals $^{2}$, and amorphous selenium ${ }^{3}$, metal halide perovskite single crystals (MHPSCs) $\mathrm{CH}_{3} \mathrm{NH}_{3} \mathrm{PbX}_{3}(\mathrm{X}=\mathrm{Cl}, \mathrm{Br}, \mathrm{Pb}$ ) are considered to be the next generation of room-temperature semiconductors for X-ray detection due to their high absorption of X-ray photons, balanced and high charge mobility-lifetime products, and the fact that they can be synthesized, deposited, and epitaxially grown by solution-processing methods inexpensively ${ }^{4-10}$. 
However, to capture hard X-ray photons ( $>100 \mathrm{keV}$ ), the thickness of the MHPSCs must be at least $1 \mathrm{~mm}$. Consequently, a long penetration path requires a large electrical field to drift the photoinduced charges for high-efficiency charge collection. However, the dark current increases with the applied bias in MHPSC-based photodiodes. Meanwhile, the noise current increases and floods the weak signal current induced by X-ray photons. Therefore, the X-ray sensitivity, response speed, and detection limit must be balanced against the noise current and dark current when setting the value of the external bias.

In addition to the crystallization quality improvements ${ }^{11-13}$, several photodiode structures have been investigated to decrease the dark current of MHPSC-based photodiodes, such as sandwiched MHPSC-based PIN photodiodes fabricated using two deposited charge-blocking layers ${ }^{14-16}$, MHPSC-based PN junctions formed by doping17,18, and MHPSC-based Schottky diodes realized using asymmetric metal electrodes ${ }^{10,19,20}$. However, most MHPSC-based PIN photodiodes use organic semiconductors ${ }^{21}$, nano-oxides ${ }^{22}$ and carbon derivatives ${ }^{23}$ as blocking layers that are not lattice-matched with MHPSCs, and the traps near interfacial layers (n-type/intrinsic and p-type/intrinsic) lead to the leakage of dark current. MHPSC-based PN junctions realized by growing n-type doped MHPSCs $\left(\mathrm{Bi}^{3+}, \mathrm{Se}^{2+}\right)$ on undoped p-type MHPSCs suffer from narrow depletion layers, rendering X-ray detection inferior compared to that with MHPSC-based PIN photodiodes ${ }^{17,18}$. MHPSC-based Schottky diodes can effectively decrease the dark current, but metals such as $\mathrm{Al}, \mathrm{Ga}, \mathrm{Ag}$, and $\mathrm{Cr}$ could react with halide ions in days due to their low work function, leading to performance degradation ${ }^{10,19,20}$. As a result, PIN photodiode structure is suitable for X-ray detectors and low traps interfaces could further decrease the noise. Thus, MHPSC-based PIN photodiodes fabricated by lattice-matched blocking layers epitaxially grown ${ }^{24-30}$ on intrinsic MHPSCs may further decrease the traps near the interfaces and lead to low dark current and noise.

In this work, we developed an MHPSC-based X-ray PIN photodiode with the structure of (ntype, bismuth-doped $\mathrm{MAPbBr}_{3}$ )/(intrinsic, $\mathrm{MAPbBr}_{2.5} \mathrm{Cl}_{0.5}$ )/(p-type, silver-doped $\mathrm{MAPbBr}_{3}$ ). The traps near the interfaces can be suppressed by solution-processed doped epitaxial growth (SPDE) grown n-type and p-type lattice-matched MHPSCs on the opposite face of the intrinsic layer. Differing from the spin-coating and evaporation fabrication strategy of nanometer-scale thickness films ${ }^{8}$, p-type and n-type layers, tens of micrometers thick, can effectively block the injected charges from the large voltage source without leakage current from pinholes. This work not only demonstrates that SPDE methods are able to fabricate high-quality PIN photodiodes for X-ray detection, but also provides an important guide to improve solutionprocessed and perovskite-based devices.

\section{Results:}




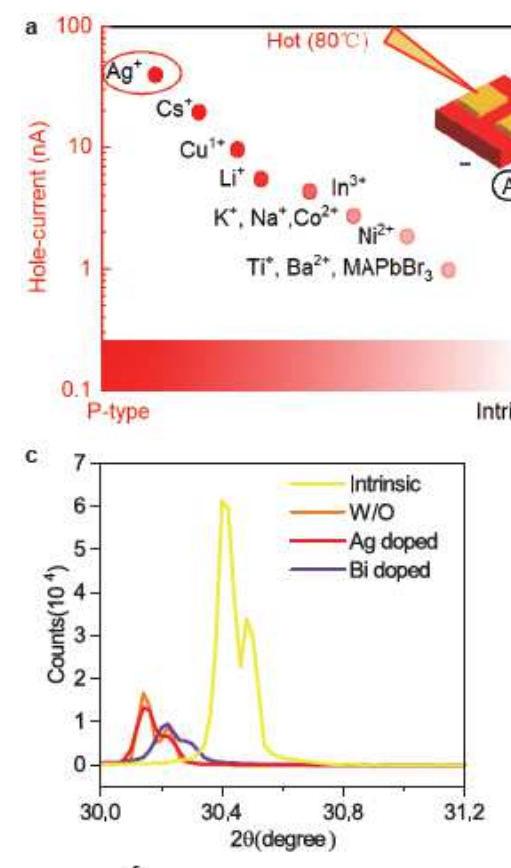

f

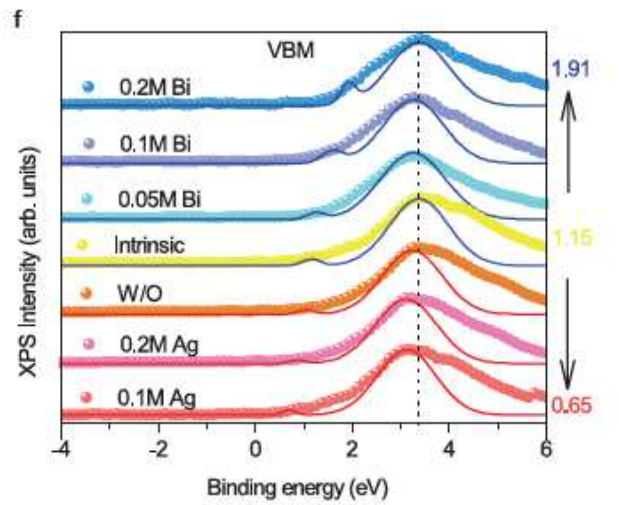

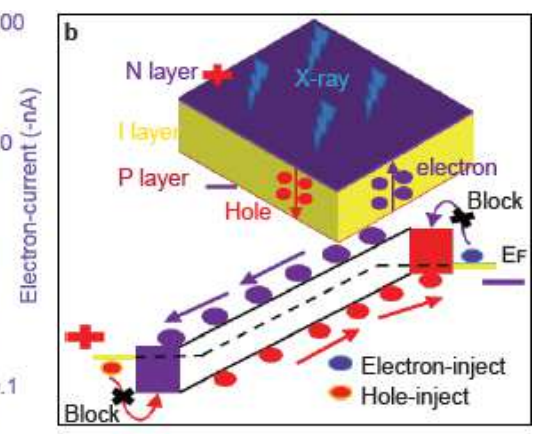
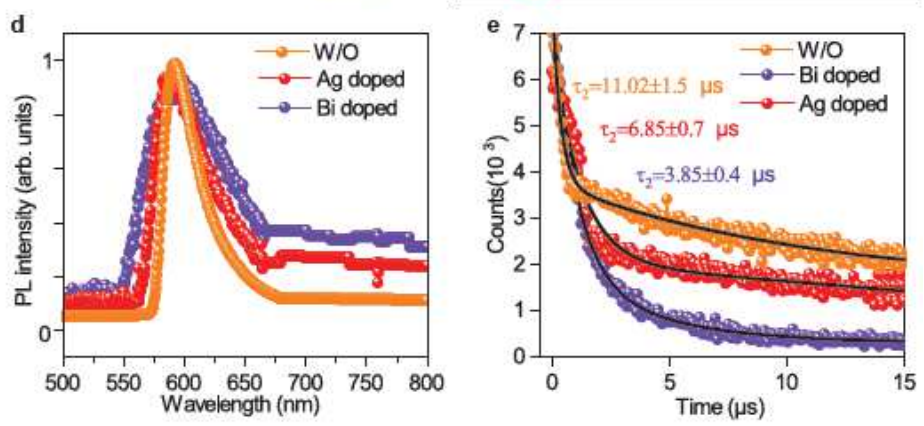

g

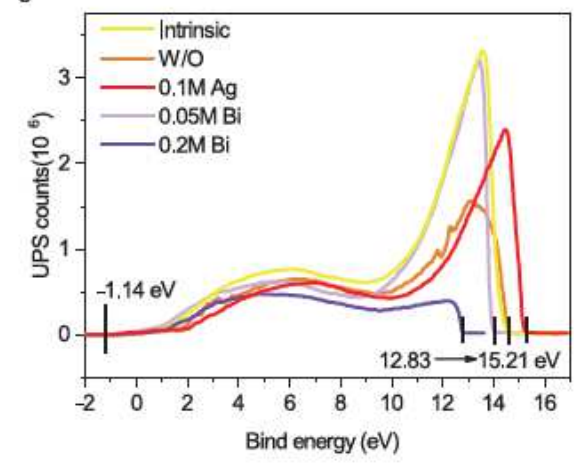

Figure 1 | Energy structure design of the X-ray PIN photodiodes. a, Thermal-probe-induced current of $\mathrm{MAPbBr}_{3}$ MHPSC with different dopants. b, Diagram of the PIN photodiodes and its work mode. $\mathbf{c}$, X-ray diffraction data for each layer from $30^{\circ}$ to $31.2^{\circ}$. d, Photoluminescence versus wavelength of intrinsic layer and doped layers. e, Minority carrier bulk lifetime of intrinsic layer and doped layers. f, VBM of each layer from $-4 \mathrm{eV}$ to $6 \mathrm{eV}$ by XPS g, UPS of each layer (using the He-I line with a photon energy of $21.22 \mathrm{eV}$ ).

To find suitable dopants, pristine $\mathrm{MAPbBr} 3$ MHPSCs were used as the substrates and metal bromide $(0.1 \mathrm{M})$ was dissolved in the precursor solution of $\mathrm{MAPbBr}_{3}$. Pristine $\mathrm{MAPbBr}_{3}$ MHPSCs were then dipped into the doped precursor for approximately $30 \mathrm{~min}$ at $70^{\circ} \mathrm{C}$. Approximately 0.2 mm-thick doped $\mathrm{MAPbBr}_{3}$ single crystals were grown on the substrates, as shown in Supplementary Fig. 1. To determine the conduction type of the doped MAPbBr 3 MHPSCs, a thermal probe method was used, as shown in the inset of Fig. 1a. Due to the temperature difference between the two probes, the majority charge carriers underwent diffusion from the hot electrode to the cold electrode, generating a diffusion current. The value of the detected current could qualitatively determine the type and magnitude of the majority charge carriers of the epitaxial doped $\mathrm{MAPBBr}_{3}$ MHPSCs. Among the 16 types of metal bromide investigated, bismuth bromide ${ }^{31-36}\left(\mathrm{BiBr}_{3}\right)$ and 
silver bromide ${ }^{37}(\mathrm{AgBr})$ are considered the most effective donors and acceptors, respectively, due to the high thermally induced diffusion current.

Based on the chosen dopants, the structure of the X-ray PIN photodiode is shown in Fig. 1b. Bidoped $\mathrm{MAPbBr}_{3}$ MHPSC and Ag-doped $\mathrm{MAPbBr}_{3}$ MHPSC are n-type and p-type layers, respectively. The intrinsic layer is made of $\mathrm{MAPbBr}_{2.5} \mathrm{Cl}_{0.5}$ MHPSCs which shows lowest traps density in MHPSCs in our previous reports ${ }^{38}$. When the X-ray PIN photodiode is employed under a large reverse bias, the $\mathrm{Bi}$-doped $\mathrm{MAPbBr}_{3}$ layer is expected to prevent hole injection from the cathode and improve the collection of photoinduced electrons. By contrast, the Ag-doped $\mathrm{MAPbBr}_{3}$ layer should stop the injection of electrons from the anode and improve the collection photoinduced holes. Compared with Schottky structures such as $\mathrm{Au} / \mathrm{CsPbBr} 3 / \mathrm{Ga}^{10}, \mathrm{Au} / \mathrm{MAPbBr} 3 / \mathrm{Ga}^{39}$ and ITO $/ \mathrm{CsPbBr}_{3} / \mathrm{Ag}^{19}$, micrometer-thick n-type and p-type layers more effectively block the injected charges. This issue is to control the quality of SPDE growth and determine a suitable doping concentration.

The ensure the quality of SPDE growth of doped MAPbBr 3 MHPSCs on a MAPbBr ${ }_{2.5} \mathrm{Cl}_{0.5}$ MHPSC (lattice constant $5.871 \AA$ ) substrate, the lattice constant is first calculated by the Bragg equation, $2 d \sin \theta=n \lambda$, where the $\lambda$ is $0.15416 \mathrm{~nm}$, from the X-ray diffraction(XRD) pattern shown in Fig. 1c. The lattice constant of the Bi-doped MAPbBr 3 MHPSC decreases from 5.925 to $5.906 \AA$ (Bidoped), while that of the Ag-doped MAPbBr3 MHPSC remains the same. The XRD data of other doping concentrations are shown in Supplementary Fig. 2. Thus, the highest mismatch rates ( $\mathrm{f}=\frac{a_{1}-a_{2}}{a_{1}+a_{2}}$, where $\mathrm{a} 1$ and $\mathrm{a} 2$ are the lattice constants of the substrate and epitaxial layer, respectively) are approximately $0.41 \%$ and $0.25 \%$ for p-type/intrinsic and n-type/intrinsic, respectively, which are good enough for epitaxial growth ${ }^{40}$

To design the energy structure of the X-ray PIN photodiode, the band gap of each layer was investigated by absorption spectroscopy, the results of which are shown in Supplementary Fig. 3. The Urbach energy ${ }^{41,42}$ is lower in pristine $\mathrm{MAPbBr}_{2.5} \mathrm{Cl}_{0.5}$ and $\mathrm{MAPbBr}_{3}$ MHPSCs than in the Bidoped and Ag-doped layers, which indicates that more traps exist inside the doped layers. The band gap remains at $2.19 \mathrm{eV}$ for the doped $\mathrm{MAPbBr}_{3}$ MHPSC as reported previously ${ }^{33,43}$. Further, the photoluminescence (PL) data for the pristine $\mathrm{MAPbBr} 3 \mathrm{MHPSC}$, Ag-doped $\mathrm{MAPbBr}_{3} \mathrm{MHPSC}$, and Bi-doped MAPbBr 3 MHPSC are shown in Fig. 1d. The peak of the PL for all remains at $592 \mathrm{~nm}$, while the full width at half maximum (FWHM) increases from $40 \mathrm{~nm}$ in in W/O MAPbBr3 MHPSC to 73 $\mathrm{nm}$ and $95 \mathrm{~nm}$ in Ag-doped MAPbBr 3 MHPSC and Bi-doped MAPbBr 3 MHPSC, respectively. The widened PL peaks indicate more traps exist inside the doped MAPbBr3 MHPSCs. In addition, the time-resolved PL of these layers was investigated, as shown in Fig. 1e. The fast component (surface carrier lifetime) increases from 345 to $937 \mathrm{~ns}$ after doping. By contrast, the slow component (bulk carrier lifetime) decreases from 11.02 to $3.85 \mu$ s after doping, which indicates that doping-induced traps inside the doped layers can accelerate the charges recombination. The traps induced by Bi play the role of dopants, as calculated in a previous report ${ }^{33}$. The Ag-doped p-type layer may be formed due to the Ag dopants, leading to a higher excess of $\mathrm{Br}$ in the MAPbBr 3 MHPSC and making the MHPSC more p-type ${ }^{37}$. 
To further investigate the band structure of n-type layers with different Bi-doping concentrations and p-type layers with different Ag-doping concentrations, the energy differences between the valence band maxima (VBM) and the Fermi level were measured by X-ray photoelectron spectroscopy (XPS). As shown in Fig. 1f, the energy differences were measured as $0.65 \mathrm{eV}, 1.15 \mathrm{eV}$, and $1.91 \mathrm{eV}$ for the $0.1 \mathrm{M} \mathrm{Ag}$-doped MAPbBr $3 \mathrm{MHPSC}$, the intrinsic layer, and 0.2 $\mathrm{M}$ Bi-doped $\mathrm{MAPbBr}_{3} \mathrm{MHPSC}$, respectively, which correspond to the p-type, intrinsic, and n-type layers. In addition, the work function was investigated by ultraviolet photoelectron spectroscopy (UPS) as shown in Fig. 1g, and $\mathrm{Bi}^{3+}$-doped $\mathrm{MAPbBr}_{3}$ MHPSC exhibited a lower work function of $7.36 \mathrm{eV}$, which indicates a better moisture stability. The higher binding energies of $\mathrm{Br}$ and $\mathrm{Pb}$ in the Bi-doped $\mathrm{MAPbBr}_{3}$ MHPSC were observed by XPS, and the profiles are shown in Supplementary Fig. 4.

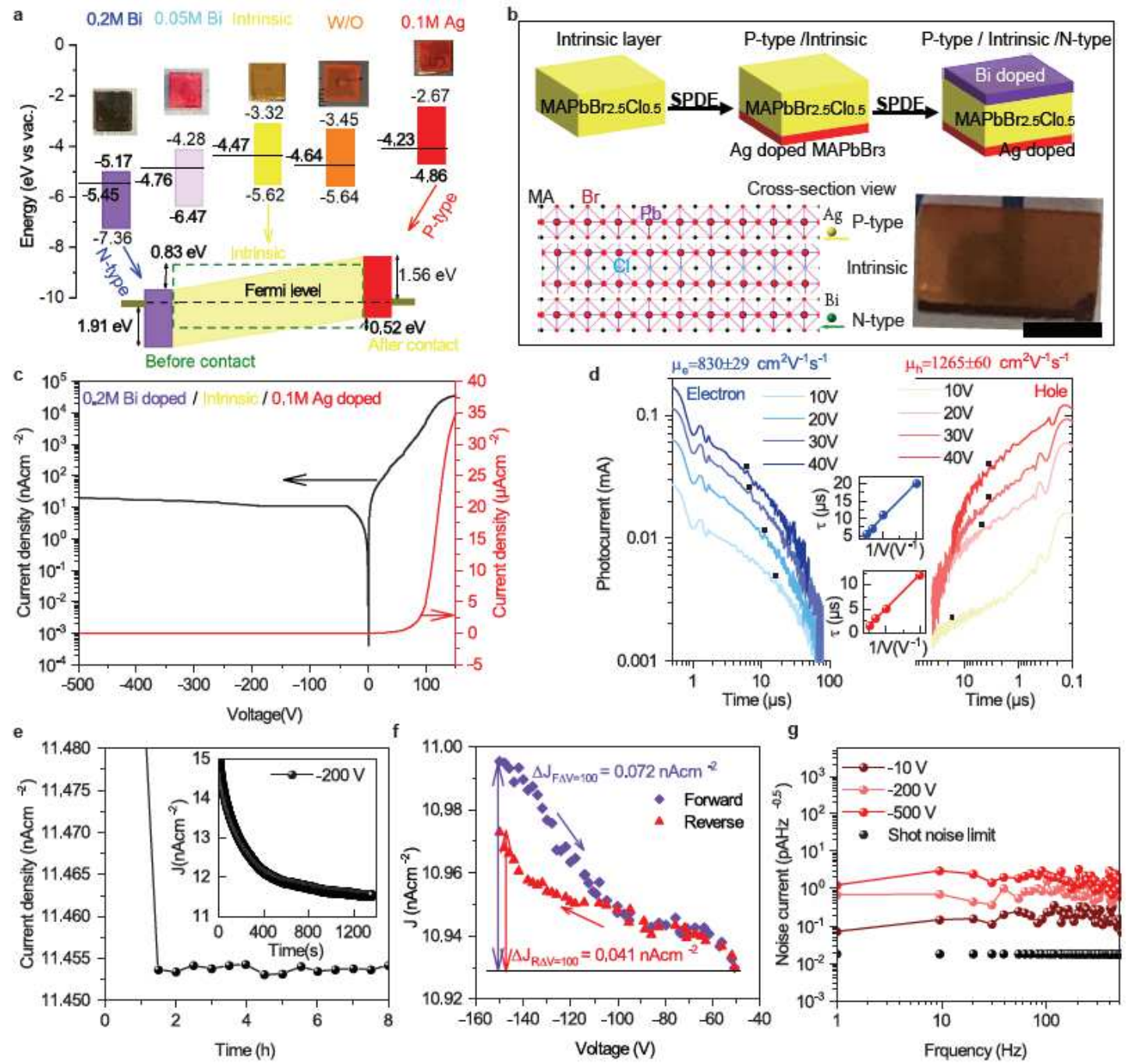

Figure 2 | Fabrication and electronic properties of the X-ray PIN photodiodes. a, Designed energy structure of the PIN photodiode. b, Fabrication process and optical images (left: cross-section view, right: top view) of the PIN photodiode $\left(6.11 \times 5.12 \times 3.91 \mathrm{~mm}^{3}\right)$, scale bar $2 \mathrm{~mm}$. c, Characterization of current density-voltage from -500 to 150 V. d, Average charge mobility of electrons (left) and holes (right) measured by TOF curves. e, Long-term 
stability of dark current under -200 V. f, Voltage bias scan from -150 V to -50 V. g, Noise current at different X-ray photodiode frequencies with varying voltage bias.

Based on the key information(Fig. 1f and Fig. 1g) of the band structure, the energy structure of these layers is shown in Fig. 2a. Owing to the low VBM $(-7.36 \mathrm{eV})$ and high conduction band minimum (CBM) (-2.67 eV) of MAPbBr 3 MHPSCs with $0.2 \mathrm{M}$ Bi and 0.1 M Ag doping concentrations, the $0.2 \mathrm{M} \mathrm{Bi}$-doped and $0.1 \mathrm{M} \mathrm{Ag}$-doped MAPbBr3 MHPSCs were chosen as n-type and p-type layers, respectively. Meanwhile, the majority-carrier densities of the p-type and n-type are calculated as $\sim 10^{14} \mathrm{~cm}^{-3}$ (holes) and $\sim 10^{19} \mathrm{~cm}^{-3}$ (electrons) by $n_{0}=n_{i} e^{\frac{\left(E_{F}-E_{i}\right)}{k T}}, p_{0}=n_{i} e^{\frac{\left(E_{i}-E_{F}\right)}{k T}}$ (where $n_{i}$ is the intrinsic carrier concentration $\sim 10^{7} \mathrm{~cm}^{-3}$, $\mathrm{k}$ is the Boltzmann constant and $\mathrm{T}$ is the temperature ${ }^{16}$. When these MHPSCs contact and the Fermi levels have the same value, the VBM difference between $\mathrm{n}$-type and intrinsic layers is obtained as $0.76 \mathrm{eV}$, while the CBM difference between p-type and intrinsic layers is $0.41 \mathrm{eV}$. Additionally, the large energy barriers of $1.91 \mathrm{eV}$ and $1.56 \mathrm{eV}$ prevent hole and electron injection.

As shown in Fig. $2 \mathrm{~b}$, the $\mathrm{MAPbBr}_{2.5} \mathrm{Cl}_{0.5}$ MHP single crystals were first grown by inverse temperature crystallization (ITC) ${ }^{6}$ for use as substrates. The bottom face of the substrate was then dipped into a $0.1 \mathrm{M} \mathrm{AgBr}$-doped $\mathrm{MAPbBr}_{3}$ solution for p-type layer SPDE growth, and the top face of the substrate was dipped into a $0.2 \mathrm{M} \mathrm{BiBr}$-doped precursor to grow the n-type layer. Finally, two gold electrodes were deposited on the opposite faces of the device. The optical photographs (Fig. 2b) of the X-ray photodiode $(6.11 \mathrm{~mm} \times 5.12 \mathrm{~mm} \times 3.91 \mathrm{~mm})$.

Fig. 2c shows the current density-voltage characteristics of the X-ray PIN photodiode from -500 to $150 \mathrm{~V}$ with a step of $2 \mathrm{Vs}^{-1}$ at $20^{\circ} \mathrm{C}$. The current density approached $61 \mu \mathrm{Acm}^{-2}$ at $150 \mathrm{~V}$, while it was approximately $11.04 \mathrm{nAcm}^{-2}$ at $-150 \mathrm{~V}$, which is typical diode behavior. The low reverse current density is attributed to the energy barriers successfully blocking the injected charges.

To investigate the charge drift process, the average mobility of electrons and holes in this X-ray PIN photodiode was measured by time-of-flight (TOF) spectroscopy, as shown in Fig. 2d because the Xray PIN photodiode made of different MHPSCs. The average electron and hole mobilities were measured as $830 \mathrm{~cm}^{2} \mathrm{~V}^{-1} \mathrm{~S}^{-1}$ and $1265 \mathrm{~cm}^{2} \mathrm{~V}^{-1} \mathrm{~s}^{-1}$, respectively, using $\tau=\frac{L^{2}}{\mu} V^{-1}$. The higher mobility measured here is approximately 4 times that of a single MAPbBr 3 MHPSC (measured by $\mathrm{Au}$ / $\mathrm{MAPbBr}_{3} \mathrm{MHPSC} / \mathrm{Au}$ for holes and Ag/n-type/ $\mathrm{MAPbBr}_{3} / \mathrm{n}$-type/Au for electrons), which indicates that the band structure improves the charge drift movements. Considering that the bulk lifetime of minority carriers is $\sim 11 \mu$ s for pristine $\mathrm{MAPbBr}_{3}$ MHPSCs, the product $\mu \tau$ is $1.39 \times 10^{-2} \mathrm{~cm}^{2} V^{-1}$.

The long-term stability of the dark current under a -200 V bias on the X-ray PIN photodiode was 
measured (Fig. 2e). It was found that ion migration inside bulk $\mathrm{MAPbBr}_{2.5} \mathrm{Cl}_{0.5}$ under bias requires approximately $1.5 \mathrm{~h}$ to achieve stability, and the dark current density drifts approximately $\sim 3$ $\mathrm{pAcm}^{-2}$, which indicates a very low noise. The stability may be attributed to the ultralow rate of charge injection. Fig. $2 \mathrm{f}$ shows the magnified forward and reverse current density-voltage from $150 \mathrm{~V}$ to $-50 \mathrm{~V}$ with step $1 \mathrm{Vs}^{-1}$. The current density varies by $0.072 \mathrm{nAcm}^{-2}$ and $0.041 \mathrm{nAcm}^{-2}$ in the forward and reverse scans, respectively. Thus, the current-density-voltage hysteresis ${ }^{44}$ is only $0.031 \mathrm{nAcm}^{-2}$ under $-150 \mathrm{~V}$ bias which is much smaller than spin-coating based PIN photodiodes and Schottky diode (Supplementary Fig. 5). Consider the same intrinsic layer, the tiny low traps density of lattice-matched interfacial layers leads to this tiny current-density-voltage hysteresis.

The noise current of this photodiode under different voltage bias was investigated and the results are shown in Fig. 2g. The noise currents are $0.11 \mathrm{pAHz}^{-0.5}(-10 \mathrm{~V}), 0.36 \mathrm{pAHz}^{-0.5}(-200 \mathrm{~V})$, and 1.47 $\mathrm{pAHz}^{-0.5}(-500 \mathrm{~V})$ at $30 \mathrm{~Hz}$. The shot noise current limit is calculated as $18-23 \mathrm{fA} \mathrm{Hz}^{-0.5}$ from the dark current. Considering that the area of this device is $31.28 \mathrm{~mm}^{2}$, the noise current is only 0.47 fA $\mathrm{Hz}^{-0.5}$ for each $100 \mu \mathrm{m} \times 100 \mu \mathrm{m}$ pixel at $-200 \mathrm{~V}$, which is comparable to those for $\mathrm{Au} / \mathrm{Cs}_{2} \mathrm{AgBiBr}_{6} / \mathrm{Au}^{45}$ and $\mathrm{Ag} /\left(\mathrm{NH}_{4}\right)_{3} \mathrm{Bi}_{2} \mathrm{I}_{9} / \mathrm{Ag}^{46}$-based low noise X-ray detectors. Such low noise current arises from the two SPDE-grown charge-blocking layers, which limit charge injection. To investigate the response speed of the X-ray PIN photodiode, the terminal capacitances are approximately $0.104 \mathrm{pF}$ and $0.074 \mathrm{pF}$ for steady and $10 \mathrm{kHz}$ (Supplementary Fig. 6), and result in a small RC product of $\sim 5$ ps (typical load resistor $50 \Omega$ ), which indicates that the response speed is mainly limited by the collection of photo generated charges. 


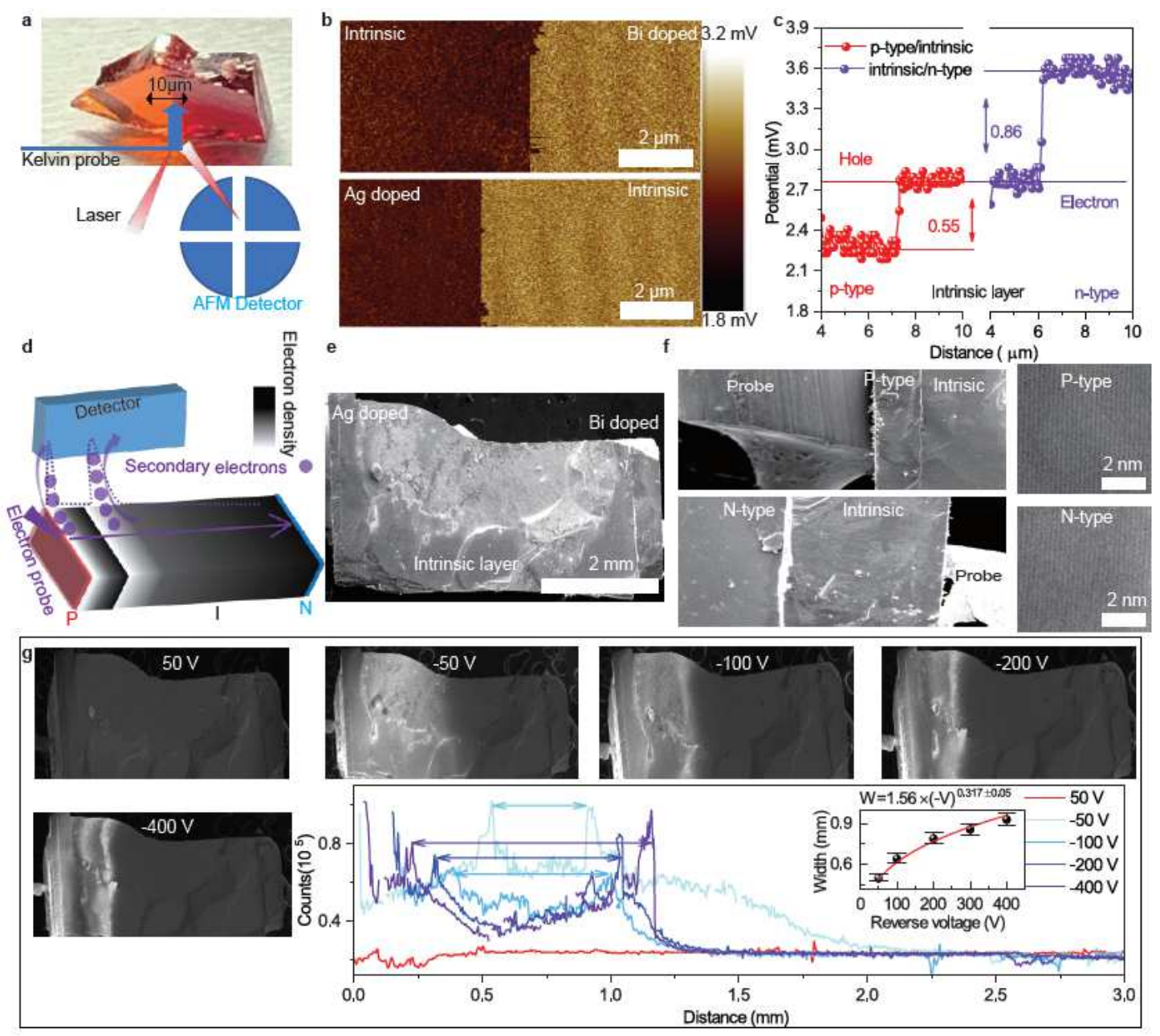

Figure 3 | Characterization of the SCR under bias. a, Illustration of the KPFM experiments. b, Contact potential difference of intrinsic/n-type(up) and p-type/intrinsic(down) interfaces. c, Line profile of the CPD. d, Illustration of the secondary electron variance with electron density in the SEM cavity. e, SEM images of the sample under 0 bias. f, Magnified cross-section of the two interfaces, HR-TEM photons of the n-type and p-type layers. g. SEM screenshots of the PIN photodiode under different voltage bias and line profile of the normalized gray value as a function of width. inset: width of the SCR verses reverses voltage.

In addition to the electrical properties, the space charge region (SCR) plays an important role in PIN junctions. Here, Kelvin probe force microscopy ${ }^{47}$ (KPFM) was used to measure the contact potential difference (CPD) between the n-type/intrinsic and p-type/intrinsic layers. As shown in Fig. 3a, the distance between the KPFM probe and the polished surface is set at $150 \mathrm{~nm}$, which is greater than the roughness of the samples of $96 \mathrm{~nm}$ and $51 \mathrm{~nm}$, respectively, as shown in Supplementary Fig. 7. Fig. $3 \mathrm{~b}$ shows the potential distribution of the scan areas, the CPDs are 0.86 $\mathrm{mV}$ and $-0.55 \mathrm{mV}$, respectively, which correlate with the energy band differences of $0.83 \mathrm{eV}$ and $0.52 \mathrm{eV}$ calculated from the XPS (Fig. 1f) and UPS profiles (Fig. 1g). Fig. 3c shows the line profile of the CPD, the SCR is located near the interfaces of n-type/intrinsic and p-type intrinsic layers. Due 
to the intrinsic layer being over 4-mm-thick, it is impossible for electrons in the n-type layer to diffuse into the p-type layer without a bias voltage. The width of the SCR in both cases is below 100 nm under illumination $(650 \mathrm{~nm})$.

In addition to the zero-bias situation, the SCR is more important when the PIN junction operates at hundreds of volts; however, it is not feasible to apply such a large bias to the KPFM system and to scan at the millimeter scale using electron beam-induced conductivity methods. There is a high charge density difference across the SCR under reverse bias, while the excited secondary electrons in scanning electron microscopy (SEM) are sensitive to the electron density of the surface. Here, a probe station is installed inside the SEM cavity (Supplementary Fig. 8) to apply voltage to the photodiode, and the SEM images can reveal the counts of secondary electrons of the photodiode surface, as shown in Fig. 3d $\mathrm{d}^{48-50}$.

Fig. 3e shows the SEM image of the X-ray PIN junction under $0 \mathrm{~V}$ bias, and when the thicknesses of the p-type, intrinsic, and n-type layers approached 80, 4200, and $200 \mu \mathrm{m}$, respectively. The p-type layer is slightly darker than the n-type layer owing to the lower electron density, and the high-resolution transmission electron microscope (HR-TEM) shows the lattice structure of the n-type and p-type layers which remains single crystalline as shown in Fig. $3 \mathrm{f}$. Typical HR-TEM photos and electron diffraction image are shown in Supplementary Fig. 9. The elemental distribution was investigated by energy-dispersive X-ray spectrometry (Supplementary Fig. 10). The screenshots of the PIN junction under $50 \mathrm{~V},-50 \mathrm{~V},-100 \mathrm{~V},-200 \mathrm{~V}$, and $-400 \mathrm{~V}$ are shown in Fig. 3g. The video of the device under sweep bias from -200 to $0 \mathrm{~V}$ is shown in the Supplementary video. When $50 \mathrm{~V}$ is applied to the PIN junction, the dark photo represents a high density of holes and electrons transported throughout the device. When under reverse bias, the dark area represents a high hole density and the bright area represents a high electron density. Owing to the external bias voltage, the SCR is located inside the intrinsic layer. As shown in the line profile in Fig. $3 \mathrm{~g}$, the width of the space charge region increases from 0.51 to $0.82 \mathrm{~mm}$ when the voltage bias increases from $-50 \mathrm{~V}$ to $-400 \mathrm{~V}$. After fitting to the equation, $W=\left[\frac{12 \varepsilon_{r(-V)}}{q \Delta n}(-V)\right]^{A}$, where $\varepsilon_{r}$ is the relative permittivity $\left(23.5 \varepsilon_{0}\right), q$ is the elementary charge $\left(1.6 \times 10^{-19} \mathrm{C}\right), \Delta n$ is the hole density $\left(10^{19} \mathrm{~cm}^{-3}\right)$, and $V$ is the applied voltage. $A$ is calculated as $0.317 \pm 0.05$, which approaches 0.33 in a linear graded junction ${ }^{51}$ which demonstrates the excited secondary electrons image can roughly measure the SCR in large thickness devices. 

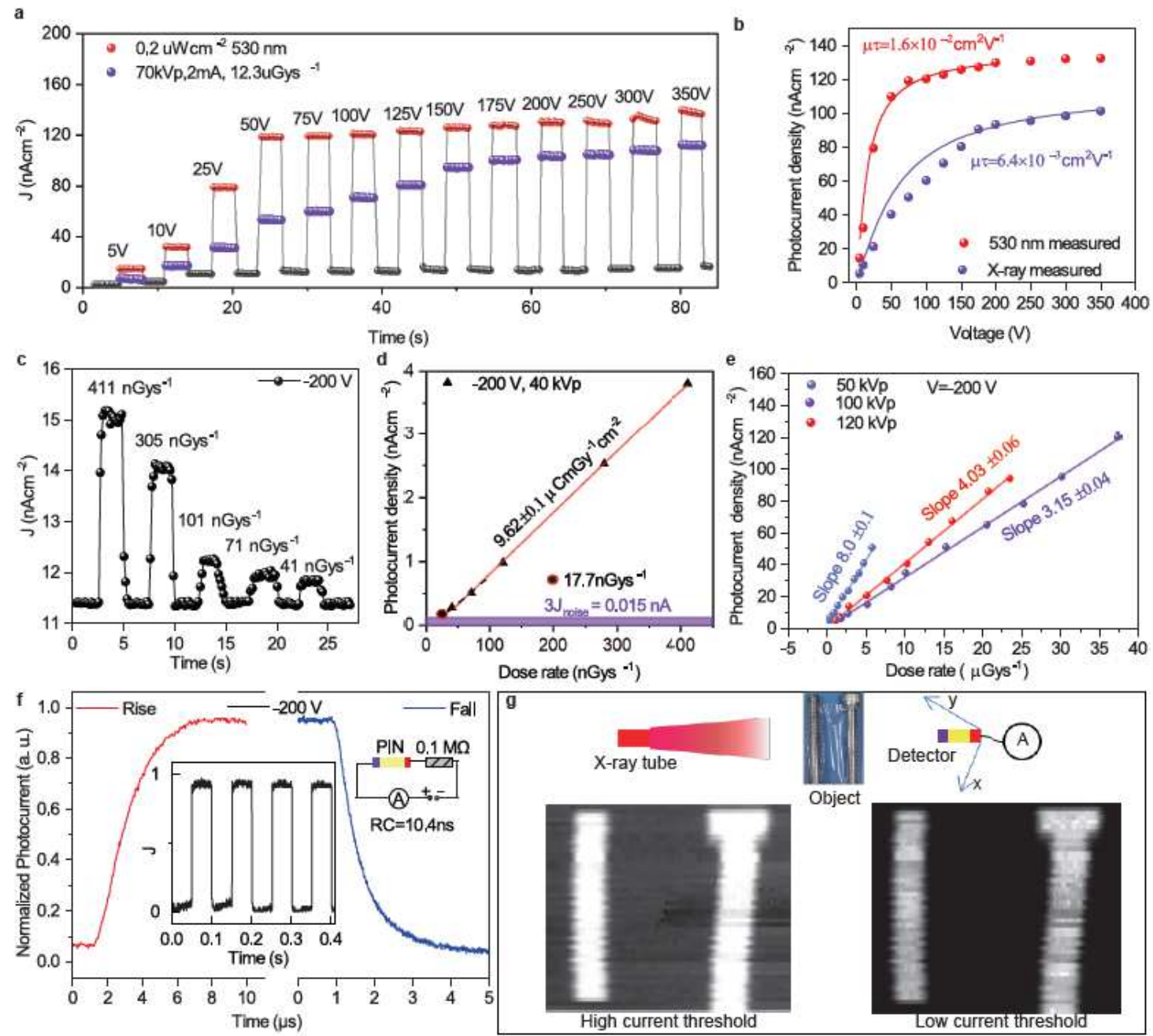

Figure 4 | X-ray detection performance and X-ray imaging. a, Photo/dark current density with different voltage bias $\mathbf{b}$, Charge collection efficiency by fitting to the Hecht equation. $\mathbf{c}$, Photo/dark current density with different dose rate $(40 \mathrm{kVp})$ under $-200 \mathrm{~V}$ bias. $\mathbf{d}, 40 \mathrm{kVp} \mathrm{X}$-ray sensitivity of the X-ray photodiode. $\mathbf{e}$, X-ray sensitivity of the Xray photodiode for X-ray photons with different photon energy. f, Response speed of the X-ray photodiode under 200 V bias. g, X-ray image captured by the X-ray photodiode.

The photodetection performance of the X-ray PIN photodiode for $70 \mathrm{kVp} \mathrm{X-ray} \mathrm{photons} \mathrm{and} \mathrm{a}$ $530 \mathrm{~nm}$ laser was first investigated. The X-ray photodiodes $\left(0.91 \times 1.12 \times 4.12 \mathrm{~mm}^{3}\right)$ show a responsivity of $0.65 \mathrm{~A} \mathrm{~W}^{-1}$ for $532 \mathrm{~nm}$ photons and X-ray detection sensitivity of $7.72 \mu \mathrm{C} \mathrm{mGy}{ }^{-1} \mathrm{~cm}^{-2}$ for X-ray photons $(70 \mathrm{kVp})$ when the applied bias swept from $-5 \mathrm{~V}$ to $-350 \mathrm{~V}$ as shown in Fig. 4a. Two different $\mu \tau$ products of $1.6 \times 10^{-2} \mathrm{~cm}^{2} V^{-1}$ and $0.64 \times 10^{-2} \mathrm{~cm}^{2} V^{-1}$ are derived from the $530 \mathrm{~nm}$ and X-ray photoconductivity curves by the Hecht equation $52,53 \frac{J}{J_{0}}=$ $\frac{\mu \tau V}{d^{2}}\left(1-e^{\frac{-d^{2}}{\mu \tau V}}\right)$, where $\mathrm{J} / \mathrm{J}_{0}$ is the charge collection efficiency, $\mathrm{J}_{0}$ is the asymptotic current density, $\mathrm{V}$ is the applied voltage, and $d$ is the thickness of the X-ray PIN photodiode, as shown in Fig. 4b. The 
large penetration depth of X-ray photons requires a strong electrical field to drift to the electrodes. Consequently, to realize high charge collection efficiency X-ray detection, large reverse is necessary and $-200 \mathrm{~V}$ is chosen as operating bias. The $\mu \tau$ product measured here is in agreement with the $\mu \tau$ product calculated from independent TOF and TRPL measurements $\left(1.39 \times 10^{-2} \mathrm{~cm}^{2} \mathrm{~V}^{-1}\right.$, Fig. 1e and Fig. 2d).

Fig. 4c shows the photocurrent response to low-dose-rate X-ray photons; the anode voltage is set at $40 \mathrm{kVp}$, while varying the anode current controls the dose rate; aluminum of thickness $2 \mathrm{~mm}$ is used as a filter. The voltage bias is set at $-200 \mathrm{~V}$ for efficient charge collection. The lowest measured dose rate from our X-ray tube is $41 \mathrm{nGys}^{-1}$ limited by the minimum anode current of our $\mathrm{X}$-ray tube ( $40 \mathrm{kVp}, 0.1 \mathrm{~mA})$, which leads to a photocurrent density of $0.28 \mathrm{nAcm}^{-2}$. The signal noise of the X-ray PIN photodiode for $40 \mathrm{kVp}$ X-ray under $-200 \mathrm{~V}$ was measured as $\sim 0.005 \mathrm{nAcm}^{-2}$. By fitting the photocurrent density versus the dose rate, the detection sensitivity for $40 \mathrm{kVp} \mathrm{X}$-ray is measured as $9.62 \mu \mathrm{C} \mathrm{mGy}^{-1} \mathrm{~cm}^{-2}$ and the lowest detectable $40 \mathrm{kVp} \mathrm{X}$-ray dose rate with signal-tonoise ratio of 3 was $17.7 \mathrm{nGys}^{-1}$ as shown in Fig. 4 d.The X-ray sensitivity of the X-ray PIN photodiode is related to the photon energy, mainly due to the different absorption coefficients. Therefore, the $\mathrm{X}$-ray detection sensitivities with $50 \mathrm{kVp}, 100 \mathrm{kVp}$, and $120 \mathrm{kVp}$ were then measured, as shown in Fig. 4e. The X-ray detection sensitivity decreases from $8.0 \mu \mathrm{C} \mathrm{mGy}{ }^{-1} \mathrm{~cm}^{-2}$ to $4.03 \mu \mathrm{C} \mathrm{mGy}^{-1} \mathrm{~cm}^{-2}$, and $3.15 \mu \mathrm{C} \mathrm{mGy}^{-1} \mathrm{~cm}^{-2}$, while the X-ray photons become harder.

In addition to the X-ray detection sensitivity, higher response speeds can shorten the exposure time, which is suitable for high frame counts and low-dose radiography. The response speed was investigated by an X-ray photodiode-external resistance $(0.1 \mathrm{M} \Omega)$ series circuit corresponding to an RC lifetime of $10.4 \mathrm{~ns}$, as shown in Fig. 4 e. The rising time (from $0 \%$ to (1-1/e)\%) and fall time (from $100 \%$ to (1/e)\%) were measured as $2.55 \mu$ s and $0.75 \mu \mathrm{s}$, respectively. The response time is comparable to the transmission time calculated from the drift movement equation, $T=\frac{L}{\mu E}=$ $0.67 \pm 0.3 \mu \mathrm{s}$. This high response speed is attributed to the high and balanced charge mobility of the intrinsic layer and the high electrical field inside the PIN photodiode. This response speed and current noise are superior to those reported in previous work ${ }^{15,16,54}$, and the X-ray sensitivity is comparable to that of spin-coating-based devices. The comparison is shown in Supplementary Table 1.

Subsequently, the X-ray PIN photodiode was used for X-ray imaging, as shown in Fig. 4f; two screws were used as the object and a two-direction slide table was used to move the X-ray PIN photodiode to realize space scanning. In the high-current-threshold image, the screw thread is clear from the background owing to the dose difference. In the low-current-threshold image, owing to the low noise current, the X-ray PIN photodiode could recognize the screw thread on the screws where the transmitted X-ray dose was extremely weak. This image suggests that this low-noisecurrent X-ray PIN photodiode could possibly realize better contrast in objects. 

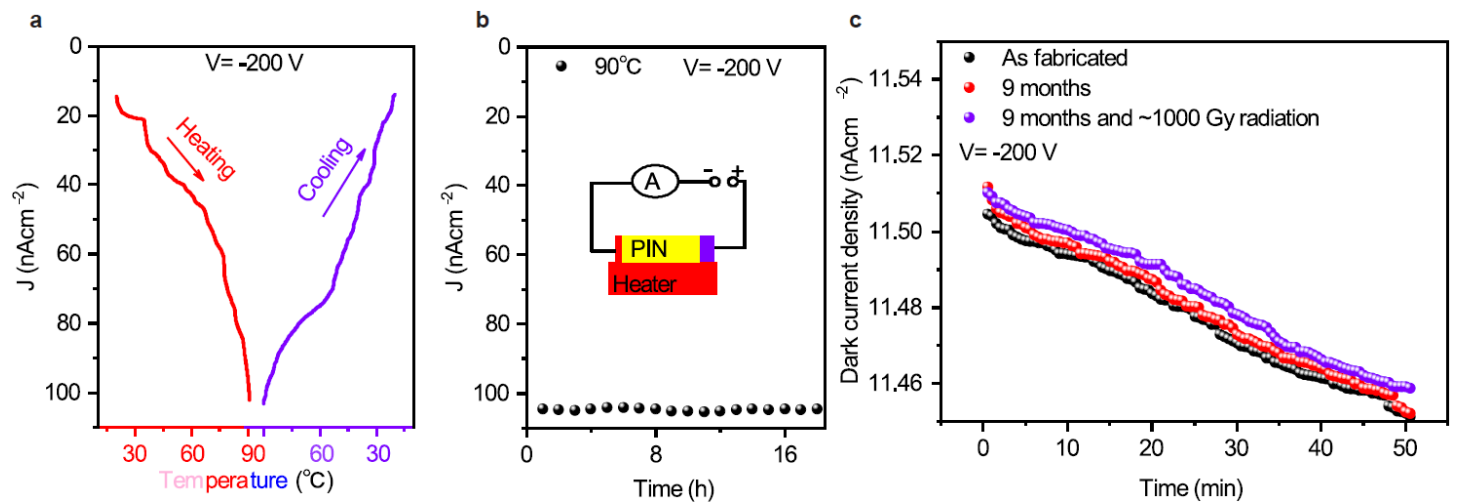

Figure 5 | Stability of the PIN photodiode. a, Thermal stability of the PIN photodiode $\mathbf{b}$, Dark current density of the PIN photodiode after 9 months and irradiation with high-dose X-rays.

In addition, ion exchange and migration ${ }^{55-58}$ may degrade perovskite-based devices; here, the device is placed at temperatures from 15 (room temperature) to $90{ }^{\circ} \mathrm{C}$ in air to accelerate this phenomenon (Fig. 5a). The dark current density increased with the temperature (nearly $1 \mathrm{nAcm}^{-2} /{ }^{\circ} \mathrm{C}$ ), indicates this device could be applied in thermal detection ${ }^{59}$. Then, the device was maintained $90{ }^{\circ} \mathrm{C}$ for $12 \mathrm{~h}$ as shown in Fig. 5b, the dark current density maintains $\sim 104 \mathrm{nAcm}^{-2}$. After that, when the device was cooled back to $15^{\circ} \mathrm{C}$ and the dark current density recovered without degradation (Fig. 5a). This proves that the ion exchange and migration speeds are extremely low in this X-ray photodiode and this X-ray PIN photodiode shows a better thermal stability than amorphous selenium. Further, as shown in Fig. 5c, the dark current density of this X-ray photodiode was investigated after 9 months in air without protection and after irradiation by high-dose X-rays in air without shielding; the drift of the dark current density is less than $0.1 \%$, which demonstrates the high stability of the X-ray PIN photodiode.

\section{Discussion}

In conclusion, the SPDE method for growing X-ray PIN photodiodes is demonstrated in this work. Based on the doping concentration and device stricture design, the X-ray PIN photodiodes exhibit remarkably low dark current and noise current under large bias. A record response time of 750 ns was achieved and a $40 \mathrm{keV} \mathrm{X-ray} \mathrm{sensitivity} \mathrm{of} 9.62 \mu \mathrm{C} \mathrm{mGy}^{-1} \mathrm{~cm}^{-2}$ was observed in this work, which could provide high-contrast and high-speed X-ray images. This work not only demonstrates that the SPDE method is capable of fabricating high-quality PIN photodiodes for X-ray detection, but also provides important insights to guide the future development of solution-processed and perovskite-based devices.

\section{References:} Detectors for Spectroscopic X-ray Imaging. IEEE Transactions on Nuclear Science 55, 572582, doi:10.1109/tns.2007.914034 (2008).

2 Sato, G. et al. Study of polarization phenomena in Schottky CdTe diodes using infrared light illumination. Nuclear Instruments and Methods in Physics Research Section A: 
Accelerators, Spectrometers, Detectors and Associated Equipment 652, 149-152, doi:10.1016/j.nima.2011.01.159 (2011).

3 Kasap, S. et al. Amorphous and polycrystalline photoconductors for direct conversion flat panel x-ray image sensors. Sensors (Basel) 11, 5112-5157, doi:10.3390/s110505112 (2011).

$4 \quad$ Wei, H. \& Huang, J. Halide lead perovskites for ionizing radiation detection. Nat Commun 10, 1066, doi:10.1038/s41467-019-08981-w (2019).

5 Zhang, M. et al. Synthesis and single crystal growth of perovskite semiconductor $\mathrm{CsPbBr}$ 3. J. Cryst. Growth 484, 37-42, doi:10.1016/j.jcrysgro.2017.12.020 (2018).

6 Saidaminov, M. I. et al. High-quality bulk hybrid perovskite single crystals within minutes by inverse temperature crystallization. Nat Commun 6, 1-7, doi:10.1038/ncomms8586 (2015).

Yakunin, S. et al. Detection of gamma photons using solution-grown single crystals of hybrid lead halide perovskites. Nature Photonics 10, 585-589, doi:10.1038/nphoton.2016.139 (2016).

$8 \quad$ Wang, X. et al. PIN Diodes Array Made of Perovskite Single Crystal for X-Ray Imaging. physica status solidi (RRL) - Rapid Research Letters 12, doi:10.1002/pssr.201800380 (2018).

Dirin, D. N., Cherniukh, I., Yakunin, S., Shynkarenko, Y. \& Kovalenko, M. V. Solution-Grown CsPbBr3 Perovskite Single Crystals for Photon Detection. Chem. Mater. 28, 8470-8474, doi:10.1021/acs.chemmater.6b04298 (2016).

$\mathrm{He}, \mathrm{Y}$. et al. High spectral resolution of gamma-rays at room temperature by perovskite CsPbBr3 single crystals. Nat Commun 9, 1609, doi:10.1038/s41467-018-04073-3 (2018). Jancik, J. et al. Microwave-Assisted Preparation of Organo-Lead Halide Perovskite Single Crystals. Cryst. Growth Des. 20, 1388-1393, doi:10.1021/acs.cgd.9b01670 (2020).

Yao, F. et al. Room-temperature liquid diffused separation induced crystallization for high-quality perovskite single crystals. Nat Commun 11, 1194, doi:10.1038/s41467-02015037-x (2020).

Wang, W. et al. Electronic-Grade High-Quality Perovskite Single Crystals by a Steady SelfSupply Solution Growth for High-Performance X-ray Detectors. Adv. Mater. 32, e2001540, doi:10.1002/adma.202001540 (2020).

14 Kim, Y. C. et al. Printable organometallic perovskite enables large-area, low-dose X-ray imaging. Nature 550, 87-91, doi:10.1038/nature24032 (2017).

15 Wei, W. et al. Monolithic integration of hybrid perovskite single crystals with heterogenous substrate for highly sensitive X-ray imaging. Nature Photonics 11, 315-321, doi:10.1038/nphoton.2017.43 (2017).

16 Wei, $\mathrm{H}$. et al. Dopant compensation in alloyed $\mathrm{CH} 3 \mathrm{NH} 3 \mathrm{PbBr} 3-\mathrm{xCl}$ perovskite single crystals for gamma-ray spectroscopy. Nat Mater 16, 826-833, doi:10.1038/nmat4927 (2017).

17 Wang, X. et al. Fabrication of Photodiodes Based on Solution-Processed Perovskite Single Crystals. IEEE Transactions on Electron Devices 66, 485-490, doi:10.1109/ted.2018.2878828 (2019).

18 Liang, F. X. et al. Fabrication of MAPbBr3 Single Crystal p-n Photodiode and n-p-n Phototriode for Sensitive Light Detection Application. Adv. Funct. Mater., doi:10.1002/adfm.202001033 (2020). 

Application. ACS Applied Electronic Materials 2, 879-884, doi:10.1021/acsaelm.9b00832 (2020).

Geng, X. et al. High-Quality Single Crystal Perovskite for Highly Sensitive X-Ray Detector. IEEE Electron Device Letters 41, 256-259, doi:10.1109/led.2019.2960384 (2020). Han, T. H. et al. Interface and Defect Engineering for Metal Halide Perovskite Optoelectronic Devices. Adv. Mater., 1803515, doi:10.1002/adma.201803515 (2019). Motti, S. G. et al. Controlling competing photochemical reactions stabilizes perovskite solar cells. Nature Photonics, doi:10.1038/s41566-019-0435-1 (2019). Chiang, C.-H. \& Wu, C.-G. Bulk heterojunction perovskite-PCBM solar cells with high fill factor. Nature Photonics 10, 196-200, doi:10.1038/nphoton.2016.3 (2016).

Chen, Y. et al. Strain engineering and epitaxial stabilization of halide perovskites. Nature 577, 209-215, doi:10.1038/s41586-019-1868-x (2020).

Tang, G. et al. Solution-Phase Epitaxial Growth of Perovskite Films on 2D Material Flakes for High-Performance Solar Cells. Adv. Mater. 31, 1807689, doi:10.1002/adma.201807689 (2019).

Kelso, M. V., Mahenderkar, N. K., Chen, Q., Tubbesing, J. Z. \& Switzer, J. A. Spin coating epitaxial films. Science 364, 166-169 (2019).

27 Jiang, J. et al. Carrier lifetime enhancement in halide perovskite via remote epitaxy. Nat Commun 10, 4145, doi:10.1038/s41467-019-12056-1 (2019).

28 Ji, L., Hsu, H. Y., Lee, J. C., Bard, A. J. \& Yu, E. T. High-Performance Photodetectors Based on Solution-Processed Epitaxial Grown Hybrid Halide Perovskites. Nano Lett. 18, 9941000, doi:10.1021/acs.nanolett.7b04445 (2018).

29 Lei, Y. et al. Controlled Homoepitaxial Growth of Hybrid Perovskites. Adv. Mater. 30, 1705992, doi:10.1002/adma.201705992 (2018).

30 Chen, J. et al. Vapor-Phase Epitaxial Growth of Aligned Nanowire Networks of Cesium Lead Halide Perovskites (CsPbX3, $X=\mathrm{Cl}, \mathrm{Br}, \mathrm{I}$ ). Nano Lett. 17, 460-466, doi:10.1021/acs.nanolett.6b04450 (2017).

31 Ulatowski, A. M. et al. Charge-Carrier Trapping Dynamics in Bismuth-Doped Thin Films of MAPbBr3 Perovskite. J Phys Chem Lett 11, 3681-3688, doi:10.1021/acs.jpclett.0c01048 (2020).

32 Zhang, R. et al. Bismuth doped lead-free two-dimensional tin based halide perovskite single crystals. Journal of Energy Chemistry 36, 1-6, doi:10.1016/j.jechem.2018.12.003 (2019).

33 Meng, R. et al. Understanding the Impact of Bismuth Heterovalent Doping on the Structural and Photophysical Properties of $\mathrm{CH} 3 \mathrm{NH} 3 \mathrm{PbBr} 3$ Halide Perovskite Crystals with Near-IR Photoluminescence. Chemistry 25, 5480-5488, doi:10.1002/chem.201805370 (2019).

34 Mosconi, E., Merabet, B., Meggiolaro, D., Zaoui, A. \& De Angelis, F. First-Principles Modeling of Bismuth Doping in the MAPbl3 Perovskite. The Journal of Physical Chemistry C 122, 14107-14112, doi:10.1021/acs.jpcc.8b01307 (2018).

$35 \mathrm{Hu}, \mathrm{Y}$. et al. Bismuth Incorporation Stabilized $\alpha$-CsPbl3 for Fully Inorganic Perovskite Solar Cells. ACS Energy Letters 2, 2219-2227, doi:10.1021/acsenergylett.7b00508 (2017). 
Zhu, F.-P. et al. Superbroad near-infrared photoluminescence from bismuth-doped CsPbl_3 perovskite nanocrystals. Opt. Express 25, doi:10.1364/oe.25.033283 (2017).

37 Siegler, T. D. et al. Addition of Monovalent Silver Cations to $\mathrm{CH} 3 \mathrm{NH} 3 \mathrm{PbBr} 3$ Produces Crystallographically Oriented Perovskite Thin Films. ACS Applied Energy Materials 2, 6087-6096, doi:10.1021/acsaem.9b01298 (2019).

Jiang, S. et al. Balance lead in solution-processed $\mathrm{CH} 3 \mathrm{NH} 3 \mathrm{PbBrxCl}(3-\mathrm{x})$ single crystals for high performance X-ray detection. Mater. Lett. 236, 26-29, doi:10.1016/j.matlet.2018.10.055 (2019).

39 Fang, Y., Dong, Q., Shao, Y., Yuan, Y. \& Huang, J. Highly narrowband perovskite singlecrystal photodetectors enabled by surface-charge recombination. Nature Photonics $\mathbf{9}$, 679-686, doi:10.1038/nphoton.2015.156 (2015).

40 Wang, X. et al. Solution-Processed Halide Perovskite Single Crystals with Intrinsic Compositional Gradients for X-ray Detection. Chem. Mater. 32, 4973-4983, doi:10.1021/acs.chemmater.9b05000 (2020).

41 Kronemeijer, A. J. et al. Two-dimensional carrier distribution in top-gate polymer fieldeffect transistors: correlation between width of density of localized states and Urbach energy. Adv. Mater. 26, 728-733, doi:10.1002/adma.201303060 (2014).

42 Sadhanala, A. et al. Preparation of Single-Phase Films of $\mathrm{CH} 3 \mathrm{NH} 3 \mathrm{~Pb}(11-x \mathrm{Brx}) 3$ with Sharp Optical Band Edges. J Phys Chem Lett 5, 2501-2505, doi:10.1021/jz501332v (2014). Nayak, P. K. et al. Impact of Bi(3+) Heterovalent Doping in Organic-Inorganic Metal Halide Perovskite Crystals. J. Am. Chem. Soc. 140, 574-577, doi:10.1021/jacs.7b11125 (2018). Kang, D. H. \& Park, N. G. On the Current-Voltage Hysteresis in Perovskite Solar Cells: Dependence on Perovskite Composition and Methods to Remove Hysteresis. Adv. Mater. 31, e1805214, doi:10.1002/adma.201805214 (2019). Pan, W. et al. Cs2AgBiBr6 single-crystal X-ray detectors with a low detection limit. Nature Photonics 11, 726-732, doi:10.1038/s41566-017-0012-4 (2017).

Zhuang, R. et al. Highly sensitive X-ray detector made of layered perovskite-like $(\mathrm{NH} 4) 3 \mathrm{Bi} 219$ single crystal with anisotropic response. Nature Photonics 14, 101-110, doi:10.1038/s41566-019-0466-7 (2019).

47 Son, J., Kim, B., Kim, C. \& Cho, J. Writing polarization bits on the multiferroic BiMnO3 thin film using Kelvin probe force microscope. Appl. Phys. Lett. 84, 4971-4973 (2004).

48 Wang, X. et al. Ultrafast lonizing Radiation Detection by p-n Junctions Made with Single Crystals of Solution-Processed Perovskite. Advanced Electronic Materials 4, doi:10.1002/aelm.201800237 (2018).

49 Seiler, H. Secondary electron emission in the scanning electron microscope. J. Appl. Phys. 54, R1-R18, doi:10.1063/1.332840 (1983).

50 Shih, A., Yater, J., Hor, C. \& Abrams, R. Secondary electron emission studies. Appl. Surf. Sci. 111, 251-258 (1997).

51 Pierret, R. F. Semiconductor Device Fundamentals. 216 (PEARSON, 2004).

52 Auden, E., Vizkelethy, G., Serkland, D., Bossert, D. \& Doyle, B. Modeling charge collection efficiency degradation in partially depleted GaAs photodiodes using the 1-and 2-carrier Hecht equations. NUCLEAR INSTRUMENTS \& METHODS IN PHYSICS RESEARCH SECTION B-BEAM INTERACTIONS WITH MATERIALS AND ATOMS 399, 12-19 (2017). 
Semeniuk, O. et al. Characterization of polycrystalline lead oxide for application in direct conversion X-ray detectors. Sci Rep 7, 8659, doi:10.1038/s41598-017-09168-3 (2017).

54 Yang, B. et al. Heteroepitaxial passivation of $\mathrm{Cs} 2 \mathrm{AgBiBr6}$ wafers with suppressed ionic migration for X-ray imaging. Nat Commun 10, 1989, doi:10.1038/s41467-019-09968-3 (2019).

55 Yuan, Y. \& Huang, J. Ion Migration in Organometal Trihalide Perovskite and Its Impact on Photovoltaic Efficiency and Stability. Acc. Chem. Res. 49, 286-293, doi:10.1021/acs.accounts.5b00420 (2016).

56 Eames, C. et al. Ionic transport in hybrid lead iodide perovskite solar cells. Nat Commun 6, 1-8, doi:10.1038/ncomms8497 (2015).

57 Zhang, Y. et al. Quantitative imaging of anion exchange kinetics in halide perovskites. Proc Natl Acad Sci U S A 116, 12648-12653, doi:10.1073/pnas.1903448116 (2019).

58 Shewmon, N. T., Yu, H., Constantinou, I., Klump, E. \& So, F. Formation of Perovskite Heterostructures by Ion Exchange. ACS App/ Mater Interfaces 8, 33273-33279, doi:10.1021/acsami.6b10034 (2016).

59 Yakunin, S. et al. High-resolution remote thermometry and thermography using luminescent low-dimensional tin-halide perovskites. Nat Mater 18, 846-852, doi:10.1038/s41563-019-0416-2 (2019).

\section{Methods:}

Materials. Lead bromide ( $\left.\mathrm{PbBr}_{2}, 99 \%\right)$ and lead chloride $\left(\mathrm{PbCl}_{2}, 99 \%\right)$ were purchased from Sigma Aldrich, USA. Methylammonium bromide (MABr) and methylammonium chloride (MACl) were purchased from Sigma Aldrich, USA. Bismuth bromide $\left(\mathrm{BiBr}_{3}, 99.9 \%\right)$, silver bromide $(\mathrm{AgBr}$, 99.9\%), and other metal bromides were purchased from Sigma Aldrich, USA. Dimethyl sulfoxide (DMSO), dimethylformamide-d7 (DMF), and $\gamma$-butyrolactone (GBL) were obtained from Aladdin. Metallic gold, silver, and gadolinium were purchased from Chinese reagent, China. All commercial products were used as received.

Fabrication of $\mathrm{X}$-ray photodiodes. The substrate $\mathrm{MAPbBr}_{2.5} \mathrm{Cl}_{0.5}$ was grown by the inverse temperature crystallization method ${ }^{6}$. Specifically, $\mathrm{PbCl}_{2}\left(0.25 \mathrm{~mol} \mathrm{~L}^{-1}\right), \mathrm{PbBr}_{2}\left(0.75 \mathrm{~mol} \mathrm{~L}^{-1}\right)$, and $\mathrm{MABr}$ $\left(1 \mathrm{~mol} \mathrm{~L}^{-1}\right)$ were dissolved in DMF $(60 \mathrm{~mL})$ and MSO $(5 \mathrm{~mL})$ mixed solution. The solutions were then filtered through a PTFE filter with a $30 \mu \mathrm{m}$ pore size. The filtrate was then transferred to a culture dish, which was placed on a programmable heating station (IKA-RET control-visc). The temperature was controlled from $40^{\circ} \mathrm{C}$ to $65^{\circ} \mathrm{C}$. For SPDE layer growth, the temperature was controlled from $60^{\circ} \mathrm{C}$ to $80{ }^{\circ} \mathrm{C}$ for the doped $\mathrm{MAPbBr}_{3}$ precursor, whereas the temperature was controlled from $60^{\circ} \mathrm{C}$ to $80^{\circ} \mathrm{C}$ for $\mathrm{MAPbBr}_{3}$. A vacuum pad was used to control the location of the substrate, and an electric-driven slide was used to control the depth of the substrate dipped in the SPDE solution. To polish and cut the additional thickness and parts of the sample, diamond powder with different sizes dissolved in diamond 
oil was used as an abrasive paste and a polishing fluid. Gold (100 nm thickness) was then deposited on the doped faces of the photodiode as electrodes, and the shape of the electrode was controlled by metal masks.

Characterization of X-ray photodiodes. X-ray diffraction patterns were obtained using an X'TRA system with a $\mathrm{Cu}$ target (Switzerland). Optical absorption spectra were measured by UV-vis spectroscopy in the range of 300 to $2000 \mathrm{~nm}$ (Lab Tech Bluestar, USA). X-ray photoelectron spectra were obtained using a PHI 5000 VersaProbe (Japan). The PL and PL decay processes were measured using a SpectraMax instrument (UK). The MHPSCs were characterized in sequence since they grow from a solution without any other process. SEM images were obtained using a Quanta 200 FEI microscope. The HR-TEM is characterized with TEM (JEM-200CX, JAPAN) for Fig. 3f. with a high voltage 220kV. The TEM photo is analyzed by ImageJ software. To avoid the influence of the surface, we broke the MHPSCs to measure the element distribution via EDX measurements. For the SCR, an FEI Nano manipulator (China) was used to apply voltage bias inside the SEM cavity. In addition, to ensure that the detectors receive enough X-ray photons in EDX, the counts per second is controlled nearly 1200 in the experiments. The KPFM probe is station (Multimode-8-AM, USA) was used to measure the CPD. Dark current density-voltage (JV) characteristics were measured using a Keithley 4200 semiconductor analyzer (USA) in the dark. The response time was measured using an Agilent oscilloscope (7.5G, USA) with a Keithley 2400 voltage source (USA) and a commercial X-ray tube operated in the pulse mode $(15 \mathrm{~mA}, 120 \mathrm{kV}, 10 \mathrm{~Hz})$ as the illumination source. The noise current was measured using an Agilent oscilloscope (USA, 7.5G) to record the voltage variations with a $100 \mathrm{k} \Omega$ load. The X-ray photodetection was performed in a lead room. The X-ray imaging system was manufactured by PERLOVE MEDICAL (China). The two-direction scanning system was custom-made.

\section{Acknowledgements}

This work is financially supported National Key Research and Development Program of China(2018YFE0125500, 2016YFB0401600), Program 111_2.0 in China(BP0719013), National Natural Science Foundation Project of China (61775034, 51879042, 61674029, 12005038), International cooperative research project of Jiangsu province (BZ2018056), Leading Technology of Jiangsu Basic Research Plan(BK20192003)

\section{Author contributions}

W. L. and X. W. supervised the project during the whole process of all experiments. X. W., Y. B., Y. P. and Y. L. grew the substrates. X. W., and Y. B., investigated the SPDE method and found suitable dopants. X. W. and Y. P. did the polish and cut process. X. W. and Y. L. did the electrical experiments. X. W. and Q. L. did the SCR measurements. X. W., J. X., J. C., E. E., D. O., J. A. and X. Z. analyzed the results. X. W. and W. L. wrote the manuscript. All authors make comments on the manuscript. 


\section{Additional information}

Correspondence and requests for materials should be addressed to W. L. or X.W.

\section{Competing financial interests}

The authors declare no competing financial interests. 
Figures
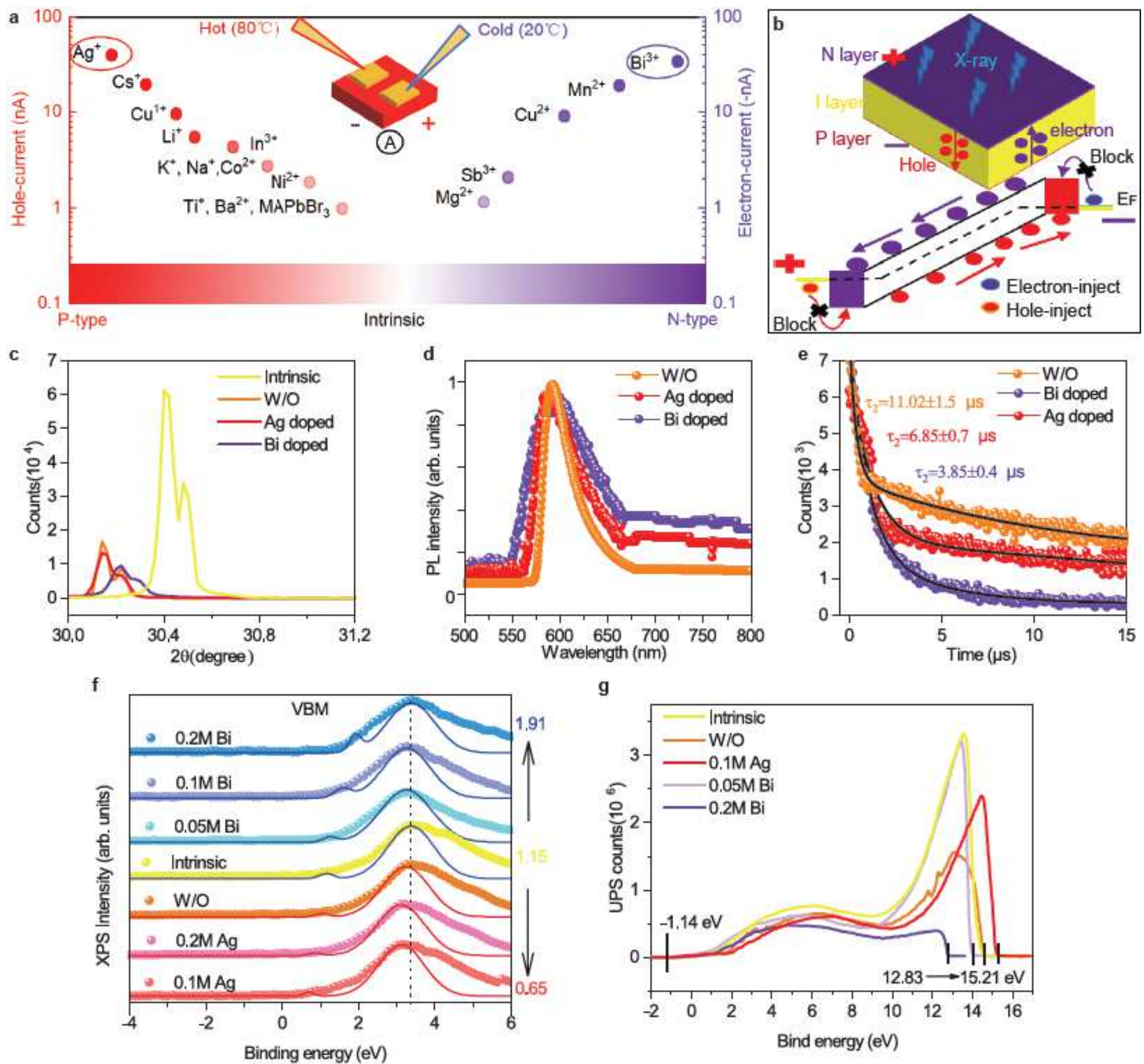

\section{Figure 1}

Energy structure design of the X-ray PIN photodiodes. a, Thermal-probe-induced current of MAPbBr3 MHPSC with different dopants. b, Diagram of the PIN photodiodes and its work mode. $c, X$-ray diffraction data for each layer from $30^{\circ}$ to $31.2^{\circ}$. d, Photoluminescence versus wavelength of intrinsic layer and doped layers. e, Minority carrier bulk lifetime of intrinsic layer and doped layers. $f$, VBM of each layer from $-4 \mathrm{eV}$ to $6 \mathrm{eV}$ by XPS g, UPS of each layer (using the He-l line with a photon energy of $21.22 \mathrm{eV}$ ). 

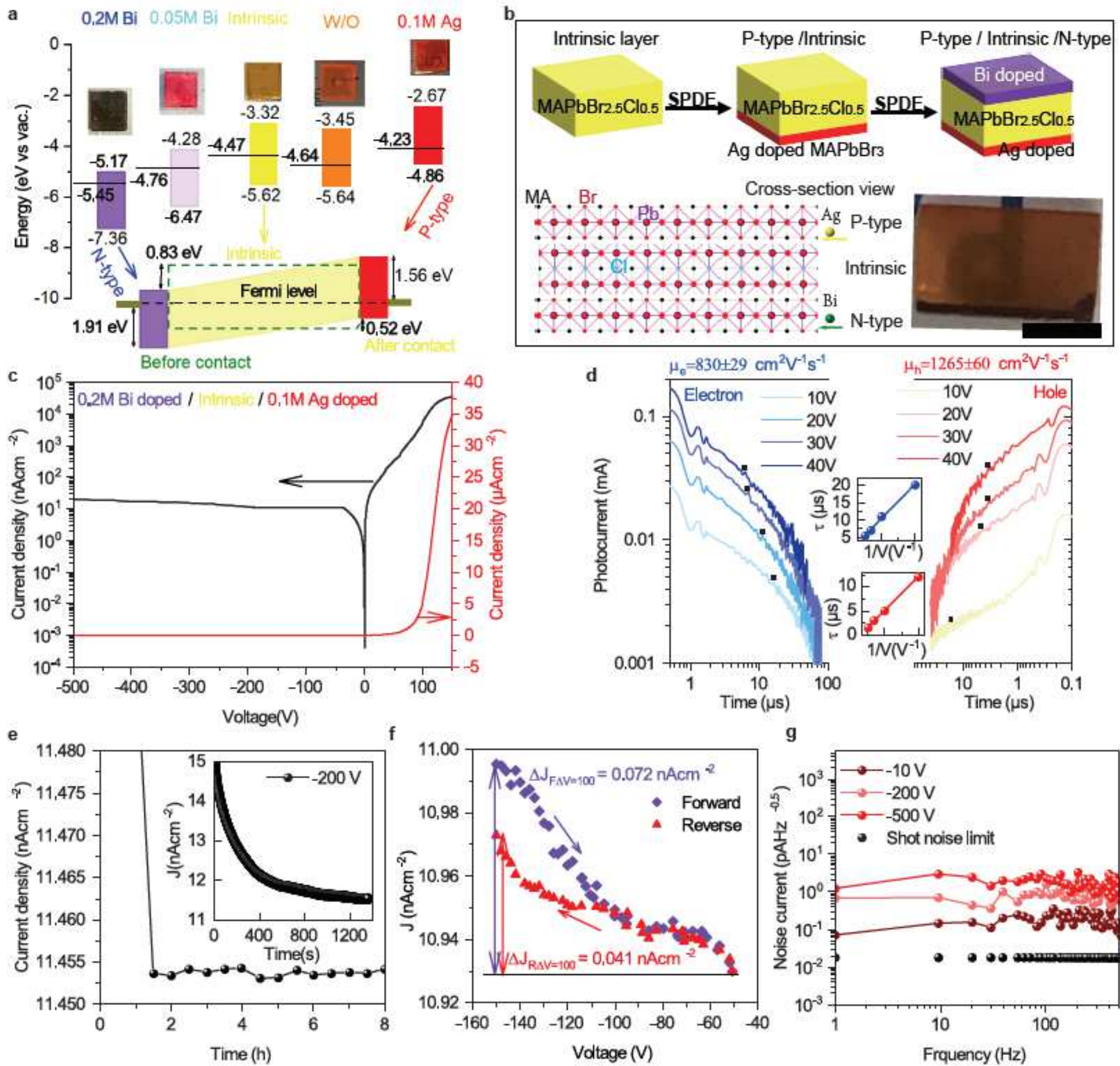

Figure 2

Fabrication and electronic properties of the X-ray PIN photodiodes. a, Designed energy structure of the PIN photodiode. b, Fabrication process and optical images (left: cross-section view, right: top view) of the PIN photodiode $(6.11 \times 5.12 \times 3.91 \mathrm{~mm} 3)$, scale bar $2 \mathrm{~mm}$. c, Characterization of current density-voltage from -500 to $150 \mathrm{~V}$. d, Average charge mobility of electrons (left) and holes (right) measured by TOF curves. e, Long-term stability of dark current under $-200 \mathrm{~V}$. f, Voltage bias scan from $-150 \mathrm{~V}$ to $-50 \mathrm{~V}$. g, Noise current at different $\mathrm{X}$-ray photodiode frequencies with varying voltage bias. 


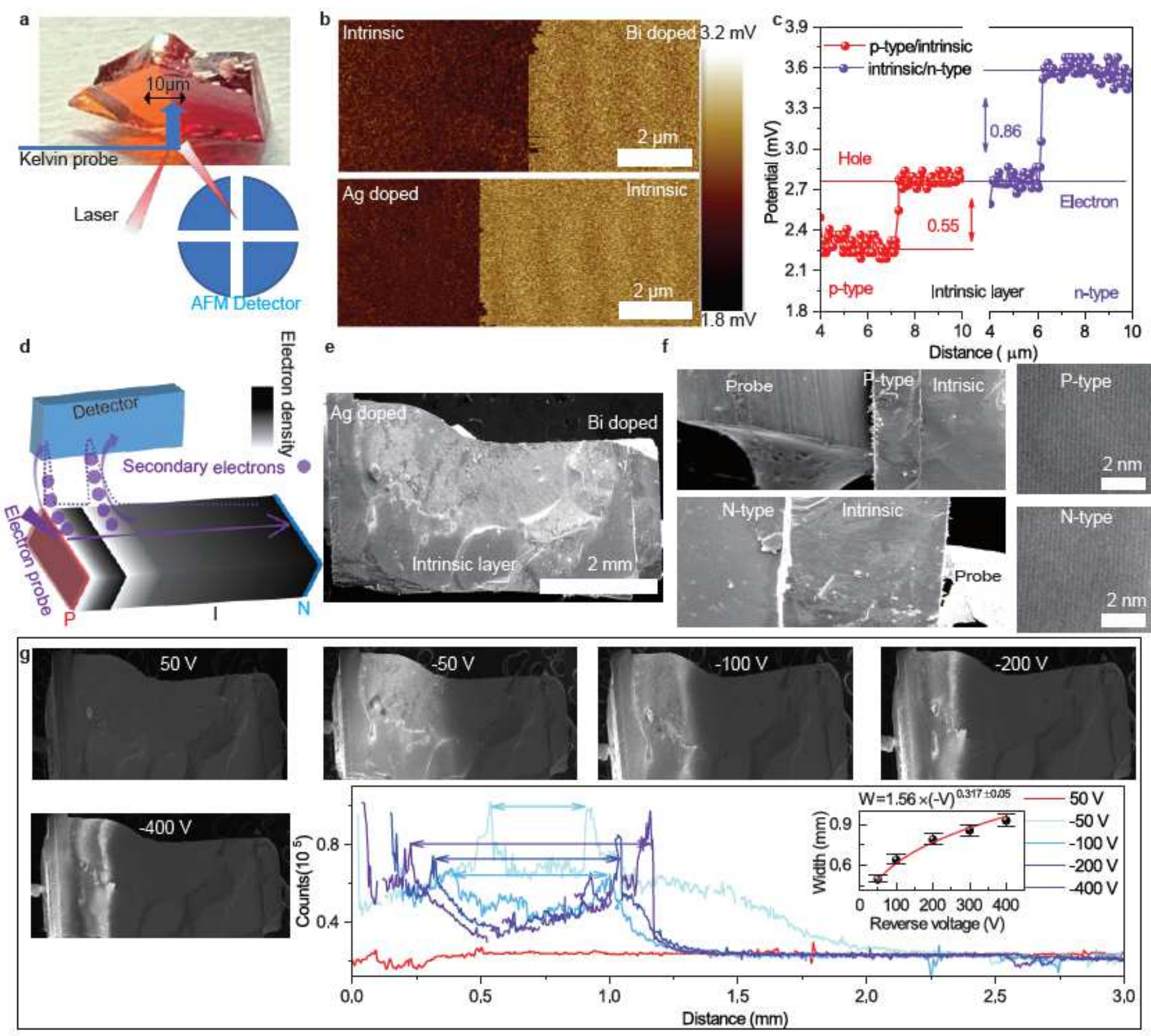

\section{Figure 3}

Characterization of the SCR under bias. a, Illustration of the KPFM experiments. b, Contact potential difference of intrinsic/n-type(up) and p-type/intrinsic(down) interfaces. c, Line profile of the CPD. $d$, Illustration of the secondary electron variance with electron density in the SEM cavity. e, SEM images of the sample under 0 bias. $\mathrm{f}$, Magnified cross-section of the two interfaces, HR-TEM photons of the n-type and p-type layers. g. SEM screenshots of the PIN photodiode under different voltage bias and line profile of the normalized gray value as a function of width. inset: width of the SCR verses reverses voltage. 
a

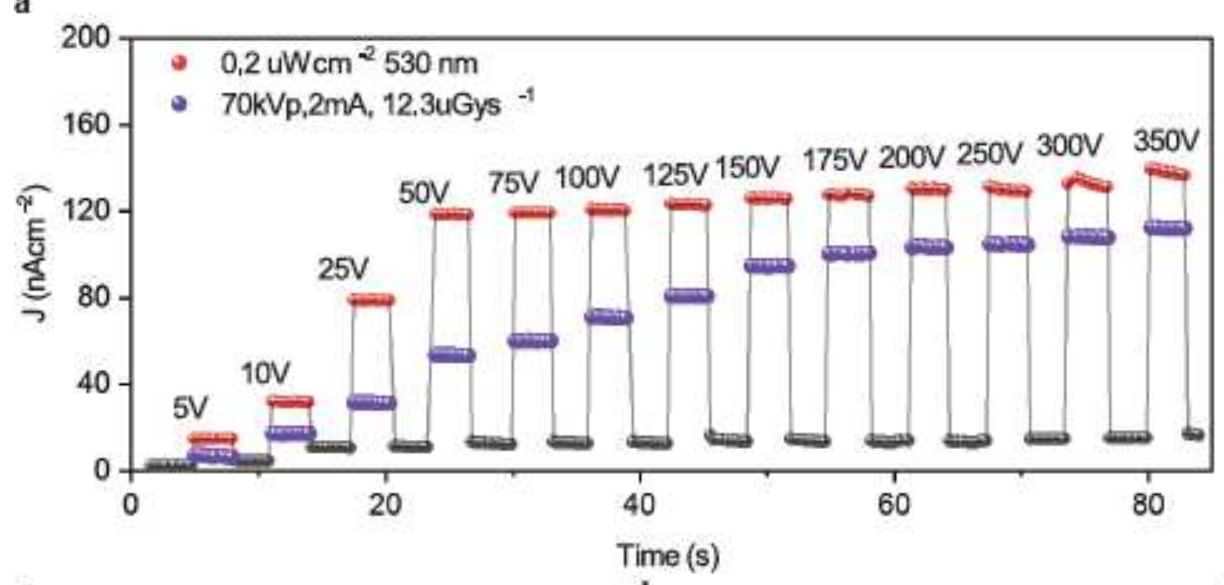

b

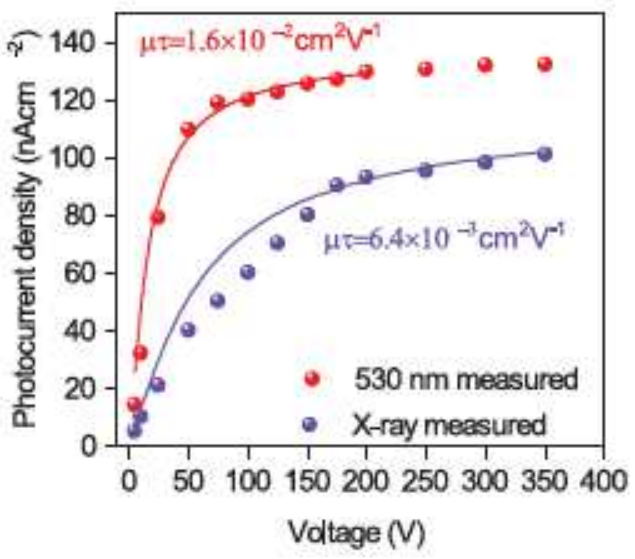

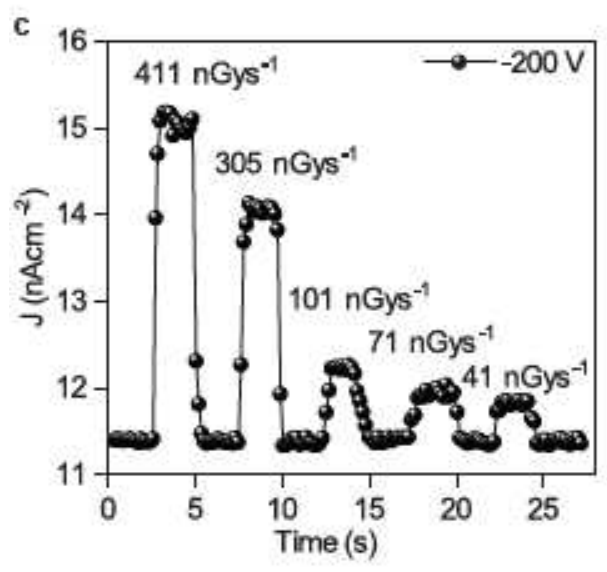
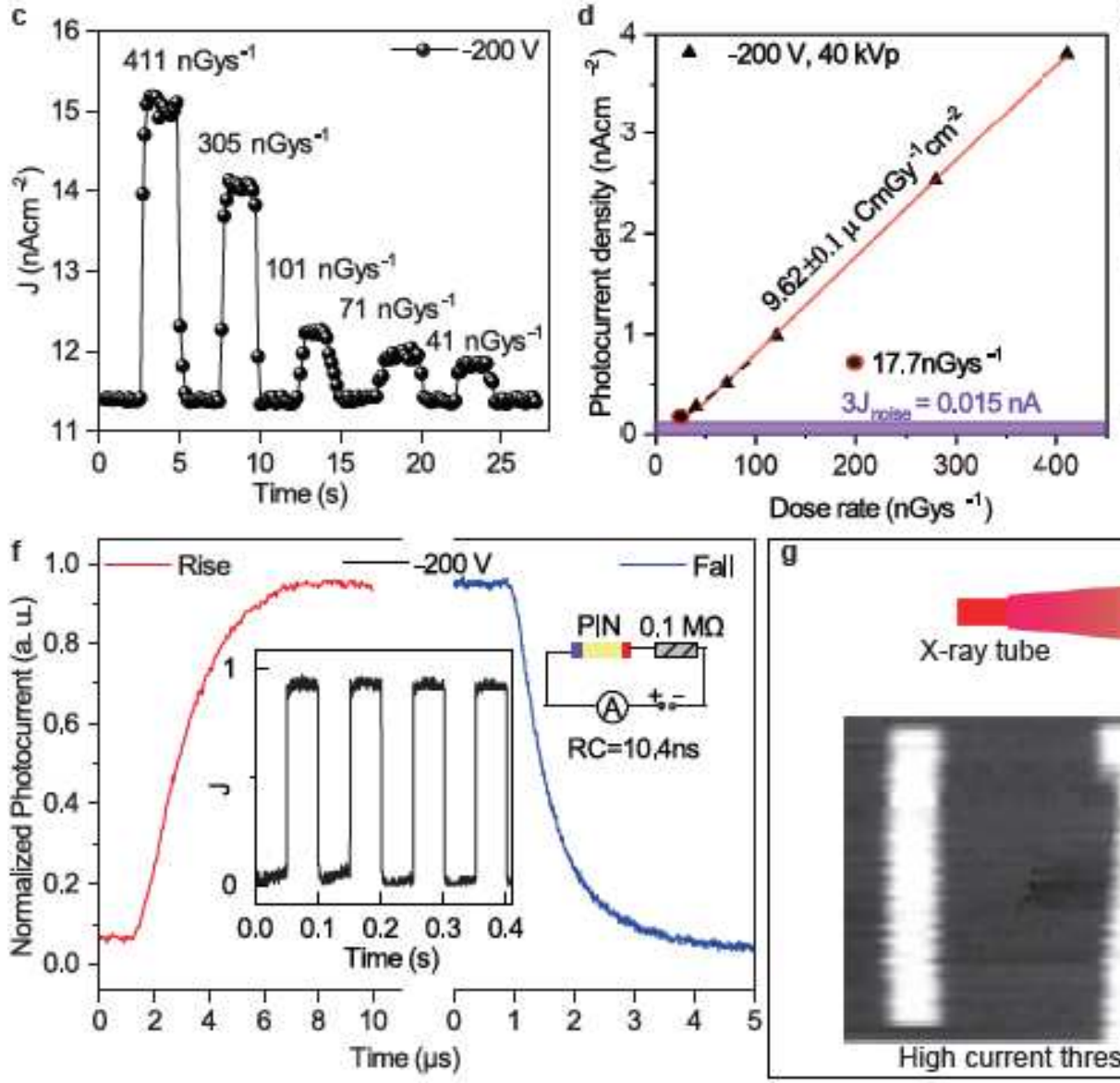

g

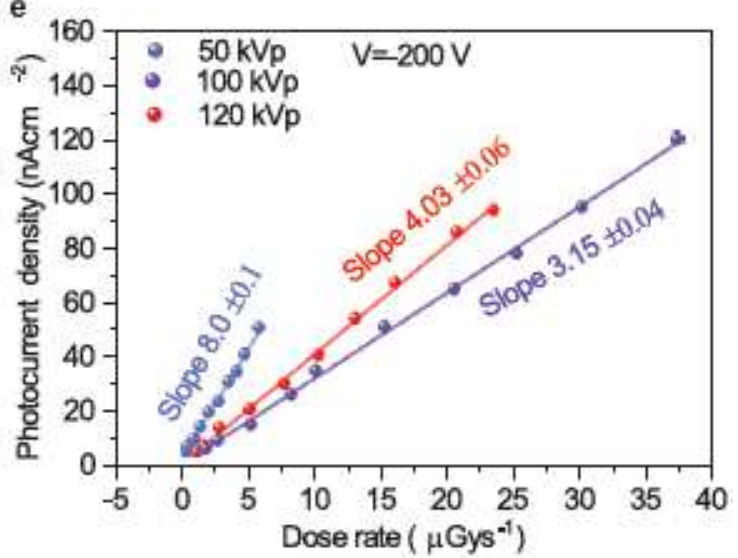

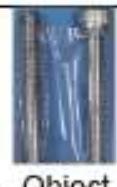

Detector
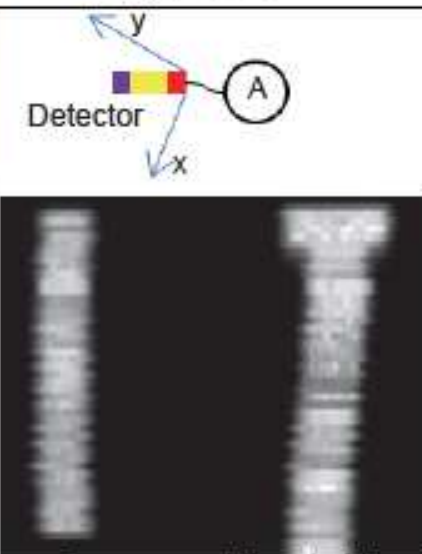

\section{Figure 4}

X-ray detection performance and X-ray imaging. a, Photo/dark current density with different voltage bias b, Charge collection efficiency by fitting to the Hecht equation. c, Photo/dark current density with different dose rate $(40 \mathrm{kVp})$ under $-200 \mathrm{~V}$ bias. $\mathrm{d}, 40 \mathrm{kVp}$ X-ray sensitivity of the X-ray photodiode. e, X-ray sensitivity of the $\mathrm{X}$-ray photodiode for $\mathrm{X}$-ray photons with different photon energy. $\mathrm{f}$, Response speed of the $\mathrm{X}$-ray photodiode under $-200 \mathrm{~V}$ bias. $\mathrm{g}, \mathrm{X}$-ray image captured by the $\mathrm{X}$-ray photodiode. 

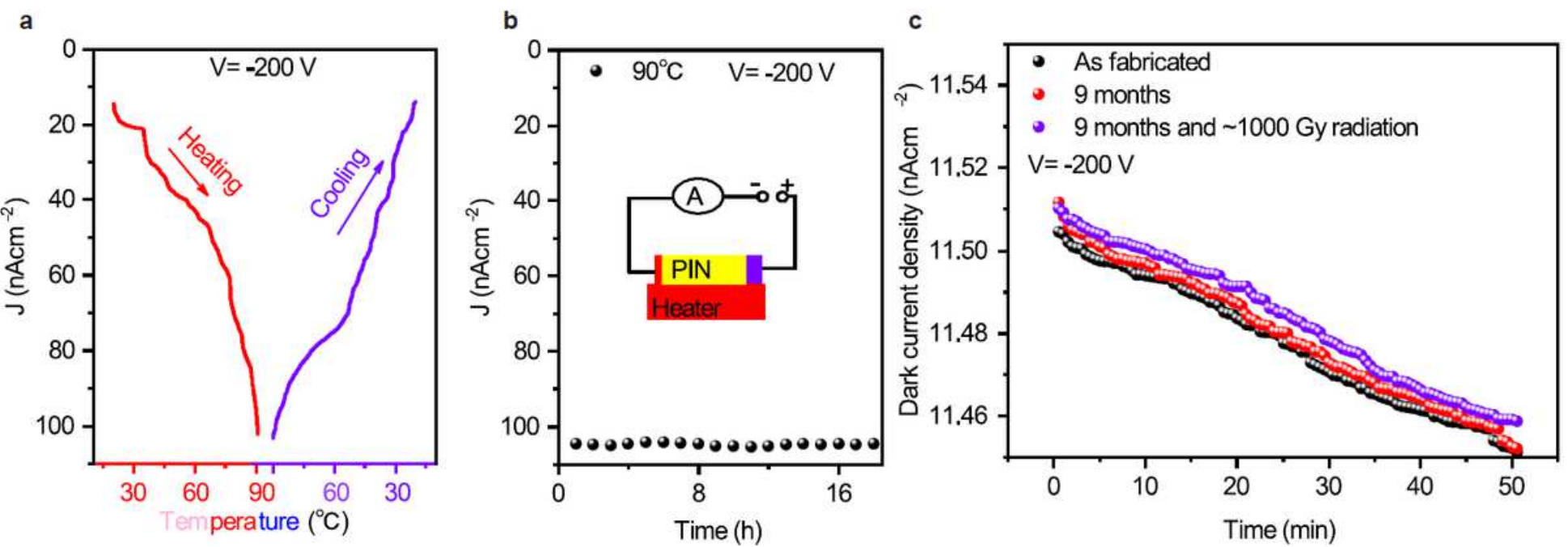

Figure 5

Stability of the PIN photodiode. a, Thermal stability of the PIN photodiode b, Dark current density of the PIN photodiode after 9 months and irradiation with high-dose X-rays.

\section{Supplementary Files}

This is a list of supplementary files associated with this preprint. Click to download.

- Supportinginformation.docx

- video.wmv 